APS

physics

This is the accepted manuscript made available via CHORUS, the article has been published as:

Effects of Complex Internal Structures on Rheology of Multiple Emulsions Particles in 2D from a Boundary Integral Method

Jingtao Wang, Jinxia Liu, Junjie Han, and Jing Guan

Phys. Rev. Lett. 110, 066001 - Published 8 February 2013

DOI: 10.1103/PhysRevLett.110.066001 


\title{
Effects of complex internal structures on rheology of multiple emulsion particles in 2-D from a boundary integral method
}

\author{
Jingtao Wang ${ }^{1,3^{*}}$, Jinxia Liu ${ }^{1}$, Junjie Han $^{1}$, Jing Guan ${ }^{2 \dagger}$ \\ ${ }^{1}$ School of Chemical Engineering and Technology, Tianjin University, Tianjin, 300072, P. R.China \\ ${ }^{2}$ School of Science, Tianjin University, Tianjin, 300072, P. R.China \\ ${ }^{3}$ State Key Laboratory of Chemical Engineering (Tianjin University)
}

\begin{abstract}
A boundary integral method is developed to investigate the effects of inner droplets and asymmetry of internal structures on rheology of 2-dimensional (2-D) multiple emulsion particles with arbitrary numbers of layers and droplets within each layer. Under a modest extensional flow, the number increment of layers and inner droplets, and the collision among inner droplets subject the particle to stronger shears. Besides, coalescence or release of inner droplets changes the internal structure of the multiple emulsion particles. Since the rheology of such particles is sensitive to internal structures and their change, modelling them as the core-shell particles to obtain the viscosity equation of a single particle should be modified by introducing the time-dependable volume fraction $\Phi(\mathrm{t})$ of the core instead of the fixed $\Phi$. An asymmetric internal structure induces an oriented contact and merging of the outer and inner interface. The start time of the interface merging is controlled by adjusting the viscosity ratio and enhancing the asymmetry, which is promising in the controlled release of inner droplets through hydrodynamics for targeted drug delivery.
\end{abstract}

PACS numbers: $83.50 . J f, 83.80 . \mathrm{Iz}, 47.11 . \mathrm{Hj}$

Multiple emulsions, which have highly ordered internal structures containing multiple layers and probably containing one or multiple smaller engulfed droplets in each layer (figure 1), have drawn much attention recently due to their great potentials in the fabrication of microcapsules applied for drug delivery systems [1]. (The particle itself is the $1^{\text {st }}$ layer; its direct daughter-droplets belong to the $2^{\text {nd }}$ layer; the droplets of the $i$-th layer have direct mother droplets belonging to the (i-1)-th layer.) The preparation of such fantastic particles is hard and highly skilful owing to their structural complexity. During the past decade, microfluidics develops rapidly and provides a great platform to generate multiple emulsions with complex but skilfully-designed internal structures [1-3]. Recently, Wang et. al. [2c] fabricated multiple emulsions containing distinct components through a hierarchical microfluidic device. The number, ratio and size of the smaller droplets in these complex particles can be precisely controlled. Droplets encapsulated in the particles can be employed as separate compartments to deliver incompatible chemicals, or as microreactors for chemical reactions. Generally, these complex particles are delivered through the flows in micro-vessels and might release their inclusions through the shell breakup under the shears. Up to now, only a few experiments have been done to investigate the rheology of double emulsions [3]. Lately, the controlled breakup of double emulsions as they flow through an orifice of a tapered nozzle was reported, and a flow regime where the inner core can be released was identified [3b, 3c]. Under the flow shears and the geometry constraint of channels, the deformed double emulsions were further converted into non-spherical microcapsules after polymerization [4a]. Chen et. al. [4b] developed a microfluidic method to carry out reactions in double emulsions by flow-controlled coalescence of encapsulated droplets, which has potentials for the fabrication of high viscosity particles and for cell assays and screening.

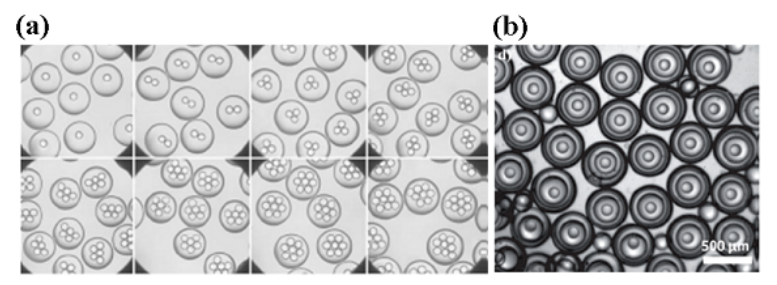

(c)

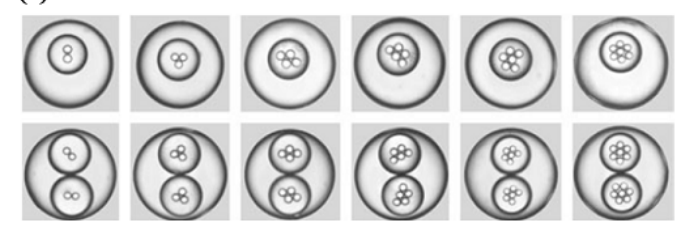

FIG. 1 Illustration of multiple emulsions particles. (a) Double emulsions containing inner droplets of various numbers. (Reproduced with permission from [2a]. Copyright 2007 Wiley-VCH Verlag GmbH \& Co. $\mathrm{KGaA}$ ) (b) Concentric multiple emulsions. (Reproduced with permission from [2b]. Copyright 2011 Wiley-VCH Verlag GmbH \& Co. KGaA) (c) Multiple emulsions with complex internal structures. (Reproduced with permission from [2a], Copyright 2007 Wiley-VCH Verlag GmbH \& Co. KGaA)

Currently, the numerical investigation of the rheology of multiple emulsions has been carried out by many groups through various methods such as level set, diffuse interface and boundary integral method et. al. [5, 6]. However, all their works are 
limited to the core-shell double emulsions. Thus, effects of complex internal structures on multiple-emulsion rheology have never been explored. In this letter, in order to investigate the effects of complex internal structures, we develop a boundary integral equation which is suitable to study multiple emulsions with orderly internal structures of arbitrary numbers of layers and droplets within each layer in microchannels with various geometries.

Figure 2 is an illustration of the deformation of a multiple-emulsion droplet under an extensional flow in a symmetric cross-slot. $r_{0}$ is the half width of the four identical arms. $S_{0}$ is the boundary including the wall, inlet and outlet of the cross-slot with normal vectors pointing inside. A two-dimensional (2D) complex particle is trapped at the stagnant point of the cross-slot and deforms under the shears of the continuous phase (CP) with viscosity $\mu$ and density $\rho$. The governing equations of the external fluid are the Stokes equations and the continuity equation

$\nabla \cdot \boldsymbol{\sigma}=-\nabla P+\mu \nabla^{2} \boldsymbol{u}=0$,

$$
\nabla \cdot \boldsymbol{u}=0
$$

where $\sigma$ is the stress tensor, $P$ is the dynamic pressure combining the pressure and gravitational terms, and $\boldsymbol{u}$ is the velocity of $\mathrm{CP}$.

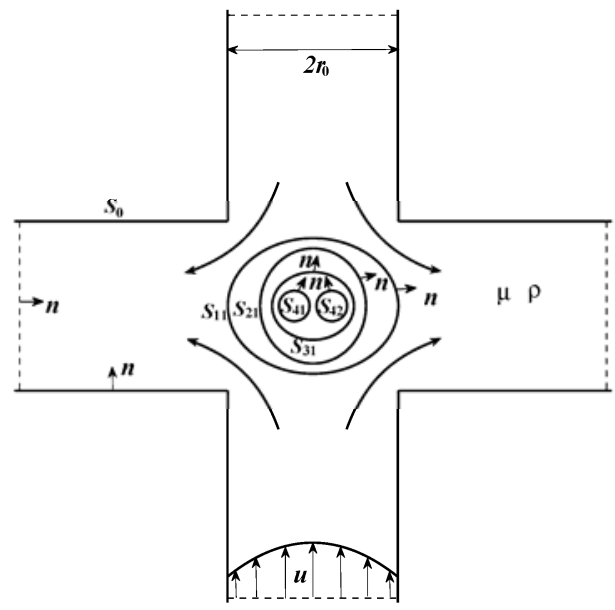

FIG. 2 Illustration of the deformation of the multiple emulsion droplet with four layers $\left(n=4, m_{1}=1, m_{2}=1\right.$, $\mathrm{m}_{3}=1, \mathrm{~m}_{4}=2$ ) in a cross-slot.

These equations can also be applied for all droplets of the multiple emulsions. Nonetheless, physical parameters in these equations must be replaced by the corresponding parameters of those droplets, such as the viscosity $\lambda_{\mathrm{ij}} \mu . \lambda_{\mathrm{ij}}$ is the viscosity ratio of the $\mathrm{ij}$-th droplet $d_{i j}$ to CP. The subscripts $i$ and $j$ indicate the $j$-th droplet of the i-th layer. The interface of the droplet with unit normal vectors $\boldsymbol{n}$ pointing outward is indicated by $S_{\mathrm{ij}}$, and its interface tension is $\gamma_{\mathrm{ij}}$. Fluid density of the ij-th droplet is $\kappa_{\mathrm{ij} \rho} \rho$, and $\kappa_{\mathrm{ij}}$ is the density ratio of the ij-th droplet to $\mathrm{CP}$. Boundary conditions (BCs) of the velocity $\boldsymbol{u}$ and surface stress $\boldsymbol{f}=\boldsymbol{\sigma} \bullet \boldsymbol{n}$ on $S_{\mathrm{ij}}$ are

$\boldsymbol{u}_{\mathrm{i}, \mathrm{j}}=\boldsymbol{u}_{\mathrm{i}, \mathrm{j}}^{\mathrm{ijmom}}$,

$$
\begin{aligned}
& \Delta \boldsymbol{f}_{\mathrm{i}, \mathrm{j}}=\boldsymbol{f}_{\mathrm{i}, \mathrm{j}}^{\mathrm{ijmom}}-\boldsymbol{f}_{\mathrm{i}, \mathrm{j}} \\
&=\gamma_{\mathrm{i}, \mathrm{j}}(\boldsymbol{\nabla} \cdot \boldsymbol{n}) \boldsymbol{n}+\left(\kappa^{\mathrm{ijmom}}-\kappa_{\mathrm{i}, \mathrm{j}}\right) \rho(\boldsymbol{g} \cdot \boldsymbol{x}) \boldsymbol{n}, \\
&\left(\mathrm{i}=1,2, \cdots \cdots, \mathrm{n} ; \quad \mathrm{j}=1,2, \cdots \cdots, \mathrm{m}_{\mathrm{i}}\right)
\end{aligned}
$$

where $\boldsymbol{g}$ is the gravity acceleration, and the superscript "ijmom" indicates the mother droplet of the ij-th droplet (when i=1, "ijmom" indicates CP). At walls $\left(S_{0}\right)$ of the cross-slot, the non-slip $\mathrm{BC}$ gives

$$
\boldsymbol{u}_{0}=0 \text {. }
$$

At the inlets and outlets $\left(S_{0}\right)$ of the cross-slot, the undisturbed flows are specified as the parabolic pressure-driven flows. The velocity profile is

$$
\boldsymbol{u}_{0}= \pm G \frac{r_{0}}{2}\left[1-\left(\frac{r}{r_{0}}\right)^{2}\right] \boldsymbol{n},
$$

where $G$ is the shear rate at the wall of inlets or outlets; positive sign is for inlets and negative sign is for outlets. The velocity at a point $\boldsymbol{x}_{0}$ on the droplet surface $S_{\mathrm{ij}}$ and outer boundaries $S_{0}$ can be described by the boundary integral equation (BIE)

$$
\begin{aligned}
L H S & =-\int_{S_{0}}[\boldsymbol{S} \cdot \boldsymbol{f}-\mu \boldsymbol{T} \cdot \boldsymbol{u} \cdot \boldsymbol{n}] d S \\
& -\sum_{\mathrm{j}=1}^{\mathrm{m}_{1}} \int_{S_{\mathrm{l}_{j}}}\left[\boldsymbol{S} \cdot \Delta \boldsymbol{f}_{1, \mathrm{j}}-\left(1-\lambda_{1, \mathrm{j}}\right) \mu \boldsymbol{T} \cdot \boldsymbol{u} \cdot \boldsymbol{n}\right] d S \\
& -\sum_{\mathrm{i}=2}^{\mathrm{n}} \sum_{\mathrm{j}=1}^{\mathrm{m}_{\mathrm{i}}} \int_{S_{\mathrm{i}_{\mathrm{j}}}}\left[\boldsymbol{S} \cdot \Delta \boldsymbol{f}_{\mathrm{i}, \mathrm{j}}-\left(\lambda^{\mathrm{ij} m o m}-\lambda_{\mathrm{i,j}}\right) \mu \boldsymbol{T} \cdot \boldsymbol{u} \cdot \boldsymbol{n}\right] d S
\end{aligned}
$$

where $L H S$ is given by

$$
\begin{aligned}
L H S & =\left\{\begin{array}{lc}
2 \pi \mu \boldsymbol{u}\left(\boldsymbol{x}_{0}\right) & \boldsymbol{x}_{0} \in S_{0} \\
2 \pi \mu\left(1+\lambda_{1, \mathrm{j}}\right) \boldsymbol{u}\left(\boldsymbol{x}_{0}\right) & \boldsymbol{x}_{0} \in S_{1, \mathrm{j}} \\
2 \pi \mu\left(\lambda^{\mathrm{ijmom}}+\lambda_{\mathrm{i}, \mathrm{j}}\right) \boldsymbol{u}\left(\boldsymbol{x}_{0}\right) & \boldsymbol{x}_{0} \in S_{\mathrm{i}, \mathrm{j}}
\end{array},\right. \\
(\mathrm{i} & =2,3, \cdots \cdots \mathrm{n}-1, \mathrm{n} \\
\mathrm{j} & \left.=1,2,3, \cdots \cdots \mathrm{m}_{\mathrm{i}}-1, \mathrm{~m}_{\mathrm{i}}\right)
\end{aligned}
$$

where $\mathrm{n}, \mathrm{m}_{1}$ and $\mathrm{m}_{\mathrm{i}}$ can be any integer. $\boldsymbol{S}$ is the fundamental solution of 2D Stokes equations and $\boldsymbol{T}$ is the associated stress kernel, which are well defined in the reference [7a]. The numerical solution of the set of boundary integral equation and boundary conditions is achieved through the 2D spectral boundary element method [7a] to get the velocity $\boldsymbol{u}$ of each point on interfaces. Then, based on known interface velocities $\boldsymbol{u}$ and stresses $\boldsymbol{f}$, the velocity of any interior point in a flow region with boundaries $B$ composed of $S_{\mathrm{ij}}$ and/or $S_{0}$ is calculated through the well-known BIE [7b],

$$
4 \pi \mu \boldsymbol{u}\left(\boldsymbol{x}_{0}\right)=-\int_{B}[\boldsymbol{S} \cdot \boldsymbol{f}-\mu \boldsymbol{T} \cdot \boldsymbol{u} \cdot \boldsymbol{n}] d B .
$$

In order to determine the evolution of the droplet shape, an explicit time-integration algorithm is employed to solve the kinematic condition $d \boldsymbol{x} / d t=\boldsymbol{u}$ at the interface. The fourth-order Runge-Kutta method are employed to lower the numerical error associated with the time integration. This numerical method has been validated by comparing our results (for concentric double emulsions) to those of Stone and Leal [6a].

Deformation parameter $D=(L-S) /(L+S)$ is employed to describe the droplet deformation, where $L$ and $S$ are the lengths of the major and the minor axes of the droplets, respectively. $U=r_{0} G / 3$ is the average velocity of $\mathrm{CP}$ at inlets, which is equivalent to capillary number 
$C a=\mu U / \gamma$ when the viscosity $\mu$ of $\mathrm{CP}$ and the surface tension $\gamma$ of the outmost interface of particles are fixed. When only one particle is considered, the radius ratio $k_{\mathrm{ij}}$ is defined as $k_{\mathrm{ij}}=r_{\mathrm{ij}} / \mathrm{r}_{11}$, where $\mathrm{r}_{11}$ is the radius of the particle. The half width $r_{0}$ of the cross-slot which equals to $2 \mathrm{r}_{11}$ is the length scale. When the volume flow rate $Q=2 r_{0}{ }^{2} / 3$, the shear rate is unit, which is selected as the scale $G_{0}$; thus, the time is scaled with the flow time scale $G_{0}{ }^{-1}$; the scale of the flow rate is $r_{0} G_{0}$.

Eqs.3-8 can treat from simple emulsions, double emulsions to multiple emulsions of any internal structures. Since the physical parameters $\lambda_{\mathrm{ij}}, \gamma_{\mathrm{ij}}$ and $\kappa_{\mathrm{ij}}$ are all disrelated for different droplets, each droplet can contain distinct components and have its own properties. However, for the common situation, i.e., only water and one oil involved, although the internal structure can still be of any pattern, the parameters are simplified to only one $\lambda$, one $\gamma$ and one $\kappa$. When effects of gravities and buoyancies are neglected, density differences vanish. In the following calculations, these simplifications are applied, and we have $U=0.333, \lambda=0.5$, and $\gamma=5$ for the fixed external flow and the fixed physical parameters.

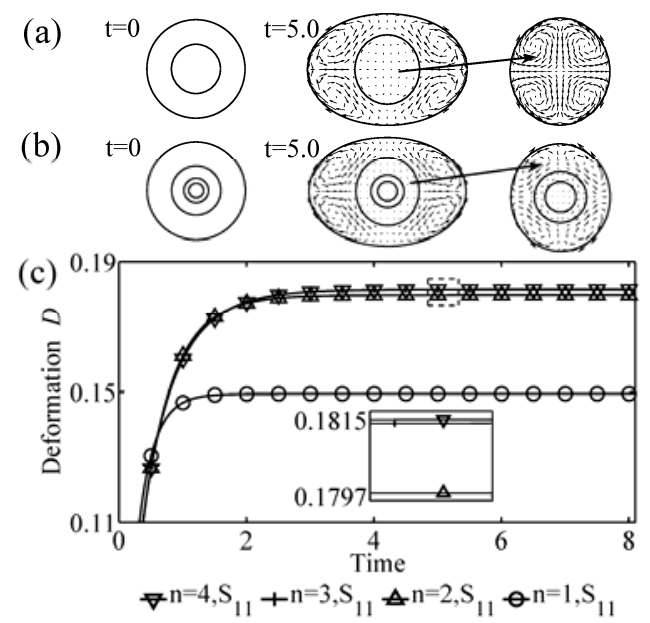

FIG. 3 Deformation of concentric multiple emulsions with layers from $n=1$ to $n=4$ in a cross-slot $\left(k_{11}: k_{21}\right.$ : $\left.k_{31}: k_{41}=1: 0.5: 0.25: 0.15\right)$. Deformation of a double emulsion (a) and a multiple emulsion with 4 layers (b) and their internal velocity fields; (c) Deformation parameter $D$ versus time for the outmost interfaces of multiple emulsions with layers up to 4 .

The deformation of concentric multiple emulsions with layers from $n=1$ to $n=4$ under a modest extensional flow is investigated as shown in figure 3 . Horizontal for $S_{11}$ and $S_{31}$ and vertical for $S_{21}$ and $S_{41}$ elongation occur in turn for the interfaces of various layers in the multiple emulsions. This alternate pattern of deformation is caused by the alternate flow fields in each layer. At the equilibrium, the deformation $D$ of the outmost interface $\left(\mathrm{S}_{11}\right)$ is apparently increased by the presence of internal layers (figure 3c), which means that the inner layers subject the particle to stronger shears.
The deformation of double emulsions containing seven inner droplets under the modest extensional flow is shown in figure $4 \mathrm{a}$ and $4 \mathrm{~b}$. "Distance" in the figure is the distance between the centre of one inner droplet and the origin, which indicates the movement of the inner droplets. When three inner droplets locate initially on the horizontal axis (figure $4 \mathrm{a}$ ), $D$ has a slight rise after it reaches an equilibrium, which is caused by the collision of the three droplets. When three inner droplets locate initially on the vertical axis (figure $4 b$ ), $D$ has a slight decline after it reaches an equilibrium, which is caused by the approach and contact of droplet \#2 and \#5 to the outer interface of the particle. As studied by Leal [8a], due to the film drainage in the head-on collision, the pressure between two approaching drops is quite high. For two inner droplets moving to each other and colliding, the resistance caused by the film drainage will request more driving forces, which enhances the outer shears to which the particle is subjected. For inner droplets moving toward to the outer interface of the mother particle, the approaching will elevate the pressure of the film between the outer and the inner interface, which will sustain the top-middle and bottom-middle of the outer interface, hamper the compression of the particle, and eventually cause the decline of $D$. Nevertheless, the coalescence caused by the collision of inner droplets, and the release of inner droplets caused by the merging of inner and outer interfaces will result in the severe change of internal structures.
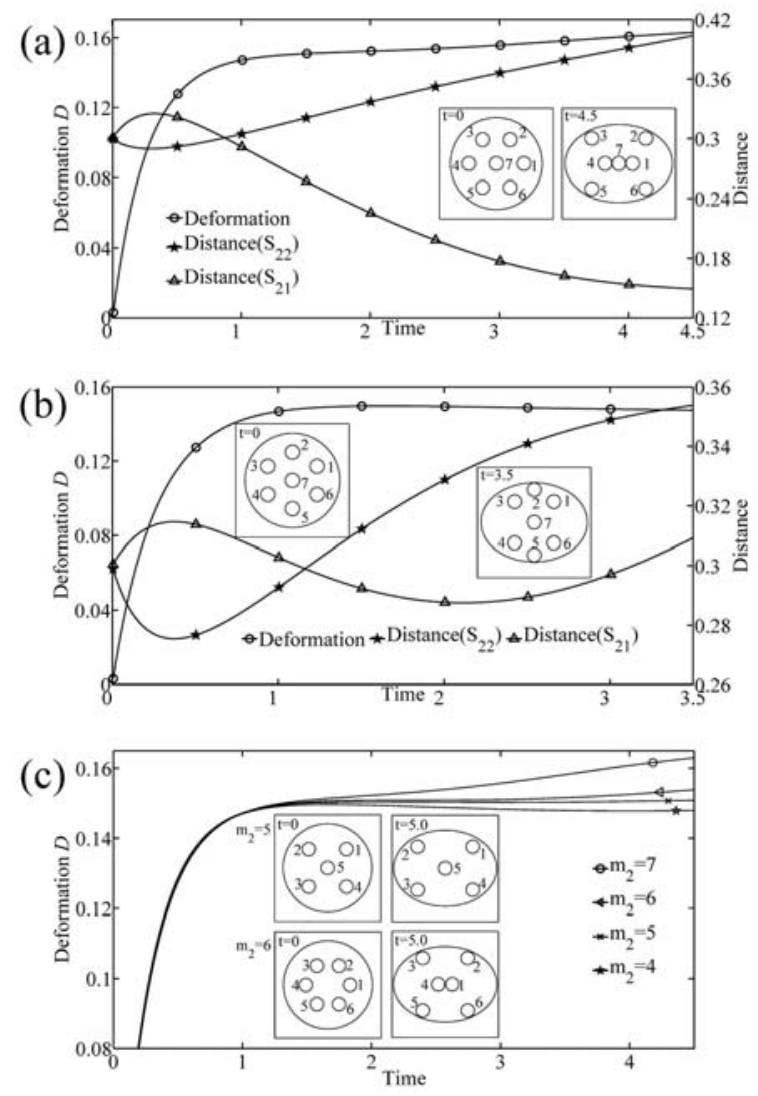

FIG. 4 Deformation of double emulsions containing seven inner droplets $\left(\mathrm{n}=2, \mathrm{~m}_{1}=1, \mathrm{~m}_{2}=7 ; k_{11}: k_{2 \mathrm{j}}=1\right.$ : $0.15, \mathrm{j}=1-7)$ and the effect of inner-droplet numbers on 
the deformation $D$. (a) Three inner droplets (\#1, 4 and 7) locate on the horizontal axis. (b) Three inner droplets (\#2, 5 and 7) locate on the vertical axis. (c) The effect of the number of inner droplets $\left(\mathrm{m}_{2}=4,5,6\right.$ and 7 ) on the deformation $D$ of the double emulsions.

Pal [5c] modelled double emulsions with multiple inner droplets as the core-shell particles with a fixed volume fraction $\Phi$ of the core to derive the formula (Eq. 10) to calculate the viscosity $\eta_{\mathrm{PE}}$ of the single particle in a dilute double emulsion,

$\eta_{\mathrm{PE}}=\lambda \mu+\left[\frac{\lambda \mu+2.5 \mu}{\lambda+1}\right] \Phi$.

When $\mu$ and $\lambda$ are fixed, $\eta_{\mathrm{PE}}$ only depends on $\Phi$ and will be constant for fixed $\Phi$. However, $\eta_{\mathrm{PE}}$ might shift under the outer flows due to the possible change of internal structures of double emulsions, which might be answered by introducing time-dependable volume fraction $\Phi(\mathrm{t})$ instead of the fixed $\Phi$.

When the number of inner droplets increases, the complication of internal structures will make the particle subject to stronger shears, as shown in figure 4c. Generally, odd number of inner droplets might cause more complexity than even number since it is easier to introduce asymmetry into the system. However, this is not always true since inner droplets of odd number might initially distribute in a symmetric pattern such as the double emulsion in figure 4 .

Deformation of multiple emulsions with an internal structure of reduced symmetry has been investigated as shown in figure 5. Although the multiple emulsion is totally symmetric about the origin (figure $5 \mathrm{a}$, time $=0$ ), the three droplets $d_{3 j}(j=1-3)$ cause the asymmetry of the particle about the horizontal axis. $S_{11}$ and $S_{21}$ reach common equilibrium shapes soon after the particle subjects to a modest extensional flow (figure 5a, time=2.0). However, the slow movement of the three small droplets leads to the asymmetry of the flow between $S_{21}$ and $S_{3 \mathrm{j}}(\mathrm{j}=1-3)$, and makes $\mathrm{d}_{21}$ move upward (figure 5a, time=11.6). Finally, $S_{21}$ and $S_{11}$ contact at the central top, which could cause the asymmetric breakup of the particle. Thus, when the triangle of $d_{3 j}(j=1-3)$ points up, the particle would break up at the central top and release the ingredient in $\mathrm{d}_{21}$ to the up-half flow field. The start time of release can be controlled by adjusting the viscosity ratio and the size of $\mathrm{d}_{3 \mathrm{j}}(\mathrm{j}=1-3)$, which is shown in figure $5 \mathrm{~d}$. $d_{\text {gap }}$ is the distance between the central top of $S_{11}$ and that of $S_{21}$. The time when $d_{\text {gap }}$ reaches 0.01 (1\% of the particle diameter, a distance within which the merging assumes to happen [8b]) is supposed when the release starts. Higher asymmetry (Large radius of $\mathrm{d}_{3 \mathrm{j}}$ enhances the asymmetry.) and lower viscosity ratios will generate the earlier release time $t_{\mathrm{r}}$ which can be calculated by the fitted formula

$$
t_{\mathrm{r}}=\left(-28.80 k_{3 \mathrm{j}}+18.18\right)+\left(-5.80 k_{3 \mathrm{j}}+2.90\right) \lambda \text {. }
$$

Due to the extreme diversity of the internal structures, there are too many multiple emulsions to be investigated. Nevertheless, based on our current analysis, the number increment of inner layers and droplets, and the collision among inner droplets will enhance the shears subjected by the particle, which might elevate the particle viscosity. However, the destabilization such as the release of inner droplets will ease the added tension of the particle, which will reduce the viscosity. Since the rheology of multiple emulsions is sensitive to the change of internal structures, the derived single-particle viscosity equation by simply modelling double emulsions containing multiple inner droplets as the core-shell particles [5c] should be modified by introducing the time-dependable volume fraction $\Phi(\mathrm{t})$. These results are also useful to the rheology response of cells with complex internal structures. Besides, complex internal structures present the possibility to design multiple emulsions for some special object. For instance, the designed asymmetric internal structures in figure 5 induce the oriented and time-controllable breakup of the particles, which provide a possibility to execute the control release of inclusions in multiple emulsions through hydrodynamics.
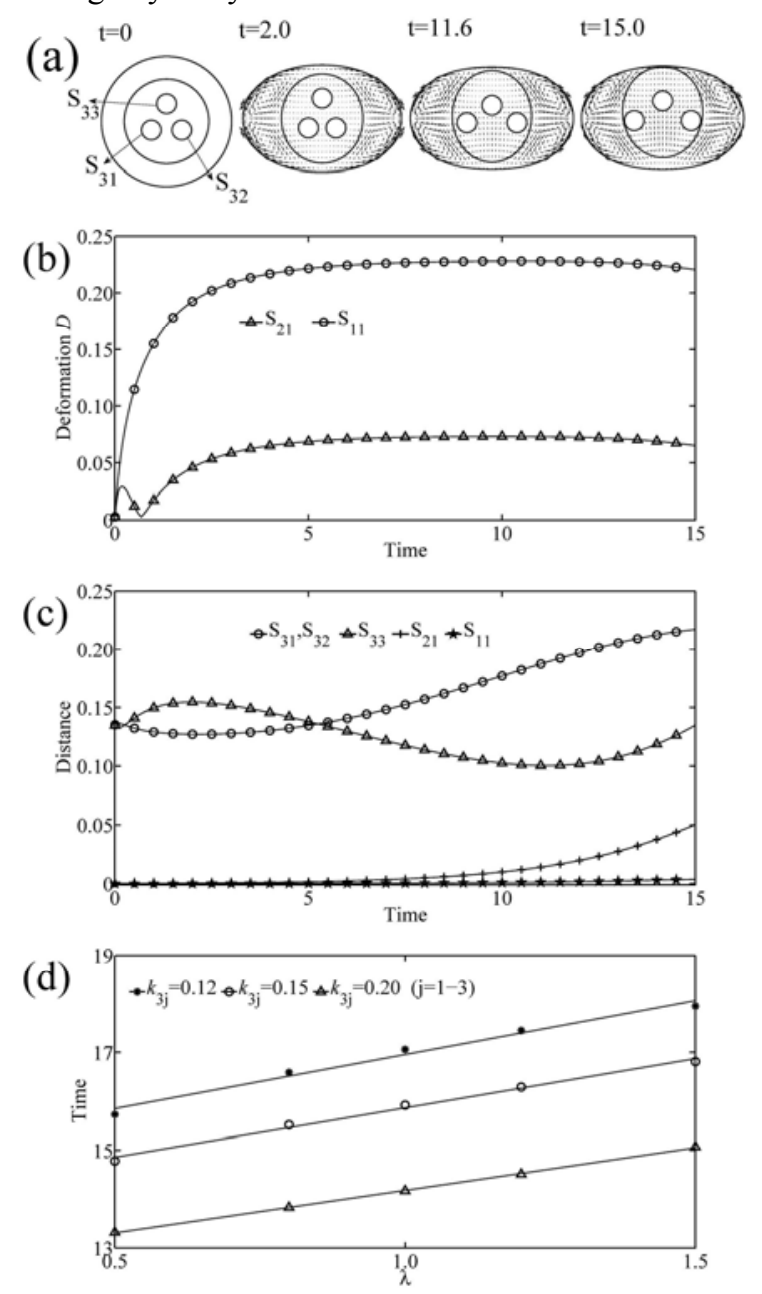

FIG. 5 Deformation of a multiple emulsion with a complex internal structures in a cross-slot $\left(n=3, m_{1}=1\right.$, $\left.\mathrm{m}_{2}=1, \mathrm{~m}_{3}=3 ; k_{11}: k_{21}: k_{3 \mathrm{j}}=1: 0.6: 0.15, \mathrm{j}=1-3\right)$. (a) Snapshots of the deformation. (b) Deformation of the interfaces of the multiple emulsions. (c) Distance of the mass centres of fluid bodies with an interface $S_{\mathrm{ij}}$ to 
the origin. (d) Effects of the viscosity ratio and the size of $d_{3 j}(j=1-3)$ on the start time of release.

This work was supported by National Natural Science Foundation of China (20806052), by Major State Basic Research Development Program of China (973 Program) (No. 2012CB720305), by General Program of Natural Science Foundation of Tianjin (11JCYBJC04300), and by National Social Science Foundation of China (09CTJ001).

*Email address: wjingtao928@tju.edu.cn
†Email address: guanjing@tju.edu.cn

[1] J. T. Wang, J. Wang, and J. J. Han, Small, 13, 1728, (2011).

[2] (a) L. Y. Chu, A. S. Utada, R. K. Shah, J. Kim, and D. A. Weitz, Angew. Chem. Int. Ed., 46, 8970, (2007); (b) S. H. Kim, and D. A. Weitz, Angew. Chem. Int. Ed., 123, 8890, (2011). (c) W. Wang, R. Xie, X. J. Ju, T. Luo, L. Liu, D. A. Weitz, and L. Y. Chu, Lab Chip, 11, 1587, (2011).

[3] (a) J. J. Ulbrecht, P. Stroeve, and P. Prabodh, Rheol. Acta, 21, 593, (1982). (b) H. S. Chen, J. Li, H. C. Shum, H. A. Stone, and D. A. Weitz, Soft matter, 7, 2345, (2011); (c) J. Li, H. S. Chen, and H. A. Stone, Langmuir, 27, 4324, (2011).
[4] (a) P. Panizza, W. Engl, C. Hany, and R. Backov, Colloids Surf., A, 312, 24, (2008). (b) H. S. Chen, Y. J. Zhao, J. Li, M. Guo, J. Wan, D. A. Weitz, and H. A. Stone, Lab Chip, 11, 2312, (2011).

[5] (a) H. C. Kan, H. S. Udaykumar, W. Shyy, and R. Tran-Son-Tay, Phys. Fluids 10, 760 (1998); (b) K. A. Smith, J. M. Ottino, M. Olvera de la Cruz, Phys. Rev. Lett., 93, 204501, (2004); (c) R. Pal, Curr. Opin. colloid Interface Sci., 16, 41, (2011); (d) Y. X. Song, J. L. Xu, and Y. P. Yang, Phys. Fluids 22, 072003 (2010); (e) C. F. Zhou, P. T. Yue, and J. J. Feng, Int. J. Multiphase Flow, 34, 102, (2008); (f) P. Gao, and J. J. Feng, J. Fluid Mech. 682, 415, (2011).

[6] (a) H. A. Stone and L. G. Leal, J. Fluid. Mech., 211, 123, (1990); (b) E. M. Toose, B. J. Geurts, and J. G. M. Kuerten, Int. J. Numer. Meth. Fluids, 30, 653, (1999).

[7] (a) P. Dimitrakopoulos and J. T. Wang, Eng. Anal. Bound. Elem., 31, 646, (2007). (b) C. Pozrikidis, Boundary integral and singularity methods for linearized viscous flow, (Cambridge University Press, Cambridge, 1992), p. 61.

[8] (a) L. G. Leal, Phys. Fluids, 16, 1833, (2004). (b) S. P. Quan, J. Lou, and D. P. Schmidt, J. Comput. Phys. 228, 2660, (2009). 